\title{
Limits on Stellar-Mass Compact Objects as Dark Matter from Gravitational Lensing of Type Ia Supernovae
}

\author{
Miguel Zumalacárregui ${ }^{1,2,3,{ }^{*}}$ and Uroš Seljak ${ }^{1,4, \dagger}$ \\ ${ }^{1}$ Berkeley Center for Cosmological Physics, LBNL and University of California at Berkeley, Berkeley, California 94720, USA \\ ${ }^{2}$ Institut de Physique Théorique, Université Paris Saclay CEA, CNRS, 91191 Gif-sur-Yvette, France \\ ${ }^{3}$ Nordita, KTH Royal Institute of Technology and Stockholm University, Roslagstullsbacken 23, SE-106 91 Stockholm, Sweden \\ ${ }^{4}$ Physics and Astronomy Department, LBNL, University of California at Berkeley, Berkeley, California 94720, USA
}

(Received 1 June 2018; revised manuscript received 14 August 2018; published 1 October 2018)

\begin{abstract}
The nature of dark matter (DM) remains unknown despite very precise knowledge of its abundance in the Universe. An alternative to new elementary particles postulates DM as made of macroscopic compact halo objects (MACHO) such as black holes formed in the very early Universe. Stellar-mass primordial black holes (PBHs) are subject to less robust constraints than other mass ranges and might be connected to gravitational-wave signals detected by the Laser Interferometer Gravitational-Wave Observatory (LIGO). New methods are therefore necessary to constrain the viability of compact objects as a DM candidate. Here we report bounds on the abundance of compact objects from gravitational lensing of type Ia supernovae (SNe). Current $\mathrm{SNe}$ data sets constrain compact objects to represent less than $35.2 \%$ (Joint Lightcurve Analysis) and $37.2 \%$ (Union 2.1) of the total matter content in the Universe, at 95\% confidence level. The results are valid for masses larger than $\sim 0.01 M_{\odot}$ (solar masses), limited by the size SNe relative to the lens Einstein radius. We demonstrate the mass range of the constraints by computing magnification probabilities for realistic SNe sizes and different values of the PBH mass. Our bounds are sensitive to the total abundance of compact objects with $M \gtrsim 0.01 M_{\odot}$ and complementary to other observational tests. These results are robust against cosmological parameters, outlier rejection, correlated noise, and selection bias. PBHs and other MACHOs are therefore ruled out as the dominant form of DM for objects associated to LIGO gravitational wave detections. These bounds constrain early-Universe models that predict stellar-mass PBH production and strengthen the case for lighter forms of DM, including new elementary particles.
\end{abstract}

DOI: 10.1103/PhysRevLett.121.141101

Introduction.-A major goal of cosmology is to characterize the physical constituents and laws of the Universe. The nature of dark matter (DM), the component sourcing the formation of large scale structure (LSS) and contributing $27 \%$ of the energy budget of the Universe [1], remains highly elusive despite decades of dedicated searches. Standard DM scenarios postulate a new elementary particle, abundantly produced in the early Universe and whose interaction with standard model particles is sufficiently suppressed, in agreement with bounds on detection experiments and collider production rates [2]. Cosmological observations are insensitive to microscopic details of DM as long it behaves as a nonrelativistic fluid, or cold dark matter (CDM), on large scales.

An alternative to microscopic dark matter scenarios invokes primordial black holes ( $\mathrm{PBH}$ ) formed in the early Universe [3-6] or other macroscopic entities, generically known as massive compact halo objects (MACHO). PBHs behave as nonrelativistic matter on large scales, making them cosmologically viable CDM candidates. They are neither detected nor produced in particle physics experiments, but can be probed by a series of small-scale effects that depend on the mass and properties of the objects [7-9]; see Fig. 3.
Interestingly, the less robust constraints on PBHs $M \sim$ 10-100 $M_{\odot}$ (solar-mass) coincide with the masses of black holes detected by the Laser Interferometer GravitationalWave Observatory (LIGO) [10,11]. This intriguing possibility lead to a revival of PBH models $[12,13]$ that could simultaneously satisfy existing bounds, provide the right dark matter abundance, and explain the high merger rate and progenitor masses inferred by the first LIGO detections. Unfortunately, uncertainties in the small-scale distribution of PBHs remain an obstacle to constrain their abundance on the basis of current gravitational wave observations alone (although see Refs. [14-19]).

Other methods are needed to reliably test the PBHDM hypothesis. In this Letter we explore the gravitational lensing predictions of PBHs-DM models for type Ia supernovae ( $\mathrm{SNe}$ ) as standard candles whose luminosity can be calibrated. Our analysis using current $\mathrm{SNe}$ data sets improves considerably on previous bounds [20]. Details of the analysis are presented in the Supplemental Material.

Magnification by compact objects.-Magnification by gravitational lensing affects the perceived SNe luminosity, which in turn modifies the inferred distance 


$$
D_{L}(z, \Delta \mu)=\frac{\bar{D}_{L}(z)}{\sqrt{1+\Delta \mu}}
$$

Here the magnification $\Delta \mu$ is defined with respect to the average (full beam) luminosity distance $\bar{D}_{L}(z)$. The probability of a given magnification is described by a probability density function (PDF) that depends in general on redshift, cosmological parameters, and the properties and abundance $\alpha \equiv \Omega_{\mathrm{PBH}} / \Omega_{M}$ of compact objects (i.e., $\mathrm{PBH}$ ) relative to the total matter density. The hypothesis of compact objects encompassing $100 \%$ of dark matter corresponds to $\alpha=\Omega_{\mathrm{CDM}} / \Omega_{M} \approx 0.844$ [1].

Compact objects induce two characteristic signatures in the magnification PDF [20-23]: (i) Most objects appear dimmer than the average, as most light bundles do not pass near any lens, shifting the maximum probability towards the empty beam demagnification and (ii) a few objects undergo significant magnification as their light bundles pass very close to one or several lenses, appearing as a tail of overluminous outliers in the PDF. The two effects are shown in Fig. 1 together with the SNe data used in the
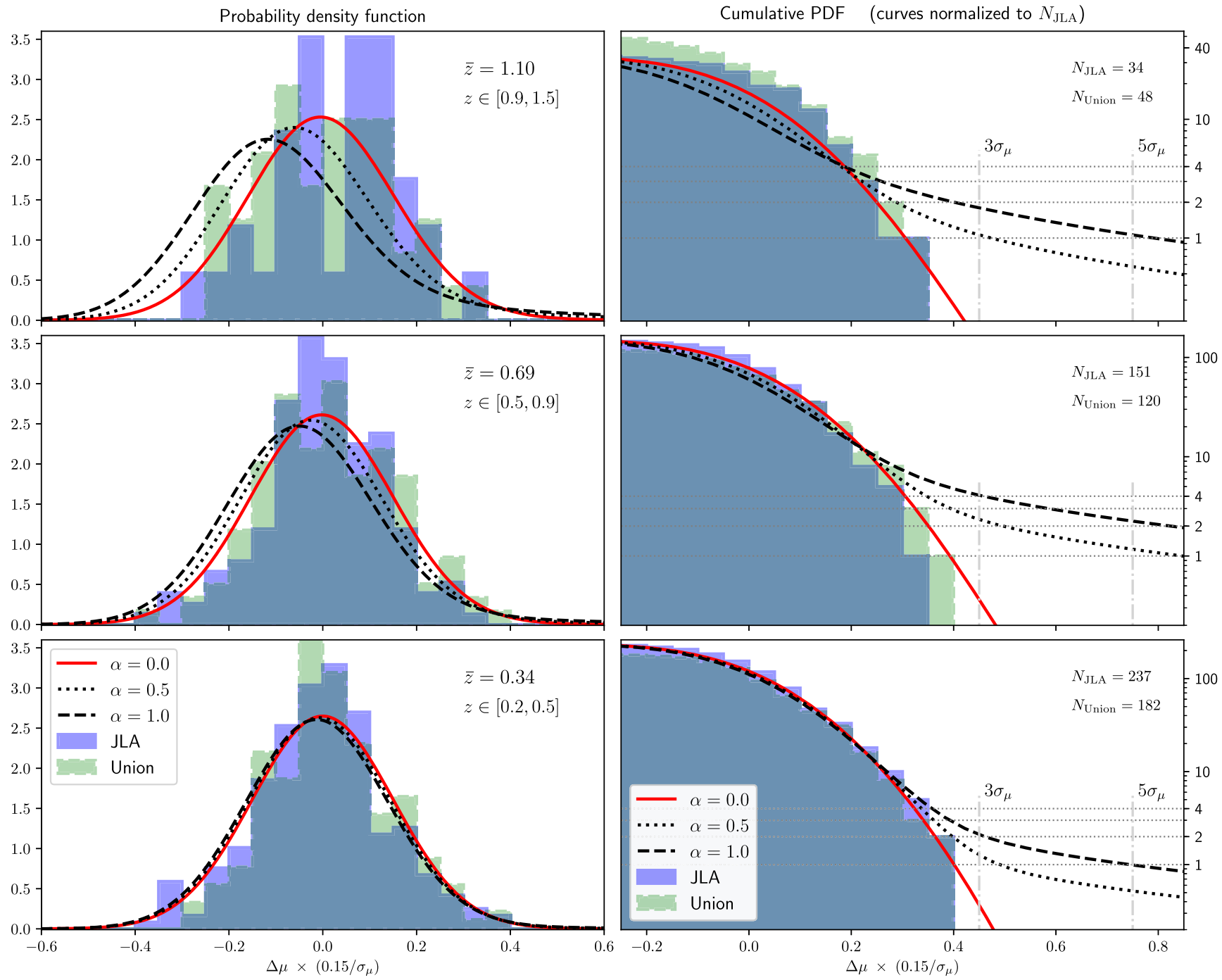

FIG. 1. Probability of lensing magnification $\Delta \mu$ [Eq. (1)] and its dependence on SNe redshift $z$ and compact object abundance $\alpha=\Omega_{\mathrm{PBH}} / \Omega_{M}$. A sizable compact object population displaces the maximum of the PDF towards the empty-beam distance, compensated with a probability tail for high magnification. Both effects grow with redshift, we show only $z \geq 0.2$. Left panel: Probability density function [Eq. (2)], normalized to unity. Histograms show to residuals of JLA (blue, solid) and Union 2.1 (green, dashed) data in the redshift ranges shown. Curves show theoretical predictions for negligible $\mathrm{PBH}$ (red, solid), 50\% PBH (black, dotted) and $\mathrm{PBH}$-only (black, dashed) universes at the mean redshift $\bar{z}$ of the subsample. A fiducial Gaussian scatter with typical SNe uncertainties $\sigma_{\mu}=0.15$ has been assumed to facilitate comparison of theory and data. Right panel: Tail distribution (cumulative) in logarithmic scale to highlight the enhanced probability of high magnification in PBH models. Histograms are normalized to the number of SNe in each redshift interval and theory predictions are normalized to the number of JLA SNe. Horizontal lines correspond to 1-4 events and vertical lines mark where $3 \sigma_{\mu}, 5 \sigma_{\mu}$ outliers are expected, relative to the SNe measurement uncertainty. 
analysis (both effects compensate since the average magnification is zero). In the limit where lenses and sources can be considered as pointlike and the mean magnification is small (so collective lensing effects such as caustic networks are negligible), the PDF is independent of the mass and distribution of compact objects [20,21]. We use a magnification PDF that combines the effects of compact objects with the distribution of cosmological LSS [23], described in the Supplemental Material [24].

The pointlike source approximation limits $\mathrm{SNe}$ lensing bounds to $M_{\mathrm{PBH}} \gtrsim 0.01 M_{\odot}$, for typical Ia SNe sizes [35]. The criterion is for the $\mathrm{SNe}$ size in the lens plane to be small compared to the Einstein radius of the lens. Since the Einstein radius grows with the lens mass, the results converge to the point-source approximation in the limit $M_{\mathrm{PBH}} \rightarrow \infty$, even for finite SNe. We explicitly computed the finite source magnification PDF for different PBH masses [36], finding an excellent agreement with the pointlike result for $M_{\mathrm{PBH}} \gtrsim 0.03 M_{\odot}$ across the magnifications relevant for the analysis (see Fig. 2). Similar computations for even smaller mass suggest that the PBH signatures might remain competitive even for values as low as $M_{\mathrm{PBH}} \sim 3 \times 10^{-4} M_{\odot}$. For a numerically efficient treatment, we define an effective lenses fraction that only counts PBHs able to magnify a given SN above a threshold based on the maximum magnification. This prescription is used to derive constraints depending on the PBH mass, as shown in Fig. 3. Our criterion is very conservative given the computation of the full PDF in Fig. 2. See Supplemental Material for details [37].

Supernovae analysis. - We will adopt a form of Bayesian hierarchical modeling for our statistical analysis and apply it to the Joint Likelihood Analysis (JLA) sample [46] and Union 2.1 [47]. The (unobservable) lensing magnification of each $\mathrm{SNe}$ is determined by a latent variable $\Delta \mu_{i}$. Rather than sampling a high-dimensional parameter space including the set $\left\{\Delta \mu_{i}\right\}$, we perform a convolution of the total lensing PDF with the intrinsic error associated to each $\mathrm{SNe}$

$L_{i}(\vec{\theta}, \alpha)=\int d \Delta \mu_{i} P_{L}\left(\Delta \mu_{i} ;, z_{i}, \alpha\right) P_{\mathrm{SNe}}\left(\Delta m_{i}, \sigma_{i}, z_{i}, \vec{\theta}\right)$

Here $P_{L}$ is the total magnification PDF (described above). $P_{\mathrm{SNe}}$ is a general non-Gaussian PDF that accounts for the intrinsic distribution of SNe luminosities and the observational noise (assumed to be a Gaussian with mean zero and variance $\left.\sigma_{i}\right)$. The quantity $\Delta m_{i}=m_{i}-\left[5 \log _{10}\left[\left(\bar{D}_{L}\left(z, \Omega_{M}\right) /\right.\right.\right.$ $\left.\mathrm{Mpc})]+25-2.5 \log _{10}(1+\Delta \mu)\right]$ is the difference between the (corrected) observed magnitude and the model prediction [Eq. (1)], including magnification. The vector $\vec{\theta}$ collectively denotes additional parameters describing the cosmology (matter fraction $\Omega_{M}$ ) and SNe population (mean magnitude, excess scatter, skewness and kurtosis, respectively, $\left.\bar{m}, k_{2}, k_{3}, k_{4}\right)$. For the JLA sample $\vec{\theta}$ includes, in addition, the $\mathrm{SNe}$ standardization parameters (stretch, color and host, respectively, $\left.a, b, \Delta_{M}\right)$. We will assume that the total likelihood is the product of individual likelihoods $L=\prod_{i} L_{i}$ and discuss correlated noise separately. We sample the space of parameters spanned by $\alpha, \vec{\theta}$, assuming a spatially flat universe with a Gaussian prior on $\Omega_{M}=$ $0.309 \pm 0.006$ [48] and the remaining cosmological parameters fixed to Planck best fit [1]. See Supplemental Material for details on the full likelihood implementation [49].

Our analysis provides stringent bounds on the compact object abundance, $\alpha<0.352$ (JLA) and $\alpha<0.372$ (Union) at $95 \%$ confidence level in the limit $M_{\mathrm{PBH}} \gg 0.01 M_{\odot}$. The PBH abundance $\alpha$ is very weakly correlated with the parameters in the $\mathrm{SNe}$ population, due mainly to the redshift dependence of the PBH signatures. In our baseline

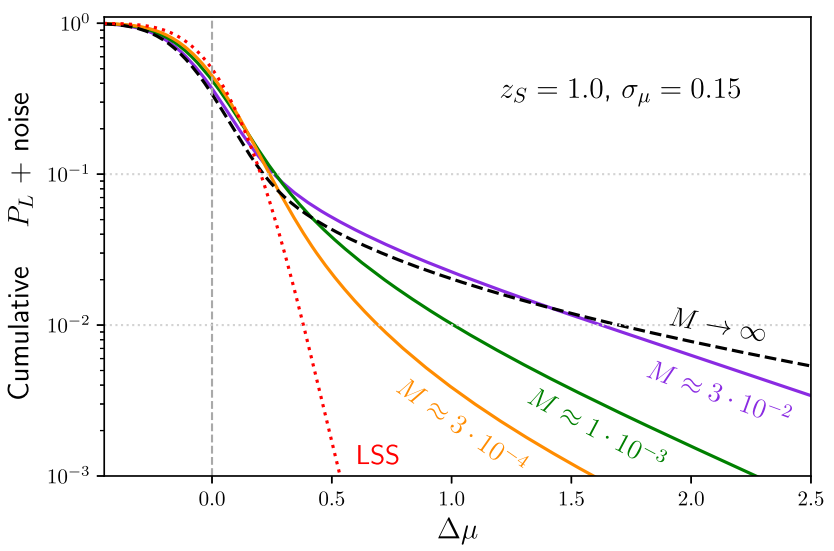

FIG. 2. Magnification PDF dependence on the PBH mass for extended SNe (compare with Fig. 1). The curves for each PBH mass assume a SNe radius $R_{S} \approx 115 \mathrm{AU}$ (see Supplemental Material, Sec. I). Right panel: PDF without noise. For $M \lesssim 10^{-2} M_{\odot}$ the result converges the analytical fit used in the analysis [21]. Note that introducing realistic noise (as in Fig. 1) would render both curves practically indistinguishable around the peak. Left panel: cumulative PDF, convolved with noise. The high-magnification tail decays faster than $(\Delta \mu)^{-3}$ but a significant fraction of highly magnified SNe are predicted even for low PBH masses. 


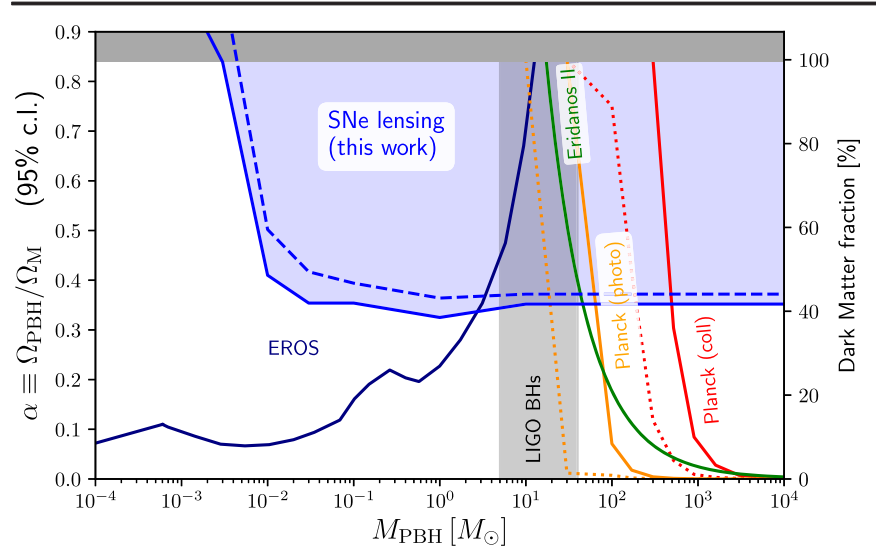

FIG. 3. Bounds on the abundance of PBHs as a function of the mass (95\% C.L.). The analysis of SNe lensing using the JLA (solid) and Union 2.1 compilations (dashed) constrain the $\mathrm{PBH}$ fraction in the range $M \gtrsim 0.01 M_{\odot}$. This range includes the masses of black hole events observed by LIGO (gray), only weakly constrained by previous data including microlensing (EROS [41]), the stability of stellar compact systems (Eridanus II $[42,43])$ and CMB $[44,45]$. The CMB excluded regions correspond to Planck-TT (solid), Planck-full (dotted) for the limiting cases of collisional (red), and photoionization (orange) (see Ref. [45] for details).

analysis the skewness (for Union) and both skewness and kurtosis are compatible with zero, suggesting that the nonGaussian SNe distribution used in the likelihood [Eq. (2)] is sufficiently general. Similar analyses fixing the compactobject mass $M_{\mathrm{PBH}}$ show how the constraints degrade, as the fraction of effective lenses reduces with decreasing mass (see Fig. 3). We note that the independence of the PDF to the specific mass distribution of compact objects makes the bounds sensitive to the total fraction in objects with masses $M_{\mathrm{PBH}} \gtrsim 0.01 M_{\odot}$. The constraints degrade slightly when the Planck + BAO prior on $\Omega_{M}$ is lifted, leading to $\alpha<$ 0.440 (JLA) and $\alpha<0.437$ (Union) at 95\% C.L., where the difference is due to a degeneracy between the empty-beam shift and the matter fraction. We note that the constraints remain competitive due to the lack of highly magnified events. See Supplemental Material for further details [53].

A potentially important systematic effect is the removal of outliers with large residuals from the base data set, as overluminous $\mathrm{SNe}$ could be either intrinsically brighter (e.g., peculiar classes, contamination) [57] or highly magnified events (e.g., due to PBHs). To address this issue we used the outliers rejected from the Union sample, noting, however, that most of those outliers have features that suggest that they are peculiar $\mathrm{SNe}$ rather than due to gravitational magnification (5/8 underluminous and $3 / 4$ overluminous). Including all the outliers from the Union sample degrades the constraints slightly to $\alpha<0.413$ (95\% C.L.). This is due to the larger abundance and significant deviations of underluminous outliers, which is better fit by a nonzero kurtosis parameter. Considering only overluminous outliers (as preferred by compact-object models, cf. Fig. 1) still results in bounds $\alpha<0.573$ (95\% C.L.). This is due to data not agreeing with the maximum magnification probability being around the empty-beam demagnification. See Supplemental Material for further details [58].

Additional analyses allowed us to establish the robustness of our results against systematic effects. We studied the impact of correlated noise using a model based on the compressed JLA likelihood with an additional free parameter. Our prescription shows that correlated noise does not have a strong effect, as it leads to only a 5\% modification of the base JLA results $(\alpha<0.363$ at 95\% C.L.). Selection bias is less problematic than in standard cosmological analysis with broad priors in $\Omega_{M}$, as the differences due to cosmology are larger than those caused by lensing. SNe population evolution across redshift may weaken the bounds similarly to lifting cosmological priors but cannot explain the lack of highly magnified SNe. See Supplemental Material for details [73].

Conclusions. - Our results on the compact object abundance reject the hypothesis of dark matter being entirely composed of stellar-mass primordial black holes at the level of $4.79 \sigma$ (JLA) and $4.54 \sigma$ (Union). The significance of the exclusion remains at the level of $2.90 \sigma$ (Union) when interpreting overluminous outliers as magnified $\mathrm{SNe}$ (despite indications that $3 / 4$ of such events are peculiar $\mathrm{SNe}$ ). Primordial black holes need to be light $\left(M_{\mathrm{PBH}} \lesssim 0.01 M_{\odot}\right.$ and hence subject to stellar microlensing bounds) or a subdominant contribution to the dark matter. Note that an extended mass function only lowers the constraints if the majority of the total mass is in the form of light $\mathrm{PBH}$.

$\mathrm{SNe}$ constraints fully cover the mass range of LIGO events and supplement other tests of macroscopic dark matter (see Fig. 3). Our analysis is complementary to stellar microlensing [41], which relies on the real-time evolution of the magnification and thus less sensitive in the limit of high PBH mass. In contrast, SNe lensing relies on the known luminosity rather than on the relative motion of lens and source, and is thus effective in the opposite limit of heavy lenses, where large Einstein radii make it more likely to produce highly magnified objects. Our results on the PBH fraction agree with recent constraints based on microlensing of quasars [74] and stars [75], caustic crossings [76-78], as well as revised estimates of LIGO event rates [16], radio and x-ray emission [79], 21-cm absorption [80], pulsar timing arrays [81], and the less conservative bounds from the cosmic microwave background $[44,45,82]$. Our constraints translate directly to other compact objects with $M \gtrsim 0.01 M_{\odot}$, e.g., Refs. [83,84].

Our analysis improves substantially on previous $\mathrm{SNe}$ lensing studies [20], reflecting the evolution of the quality and quantity of data. Larger $\mathrm{SNe}$ catalogues (e.g., Refs. [85-87]) will significantly increase the constraining power of this technique [88]. Gravitational lensing methods together with a variety of other techniques involving 
gravitational waves [16,89-92], lensing of fast radio bursts [93], astrometry [94], pulsar timing [81], and CMB $[44,45,82,95,96]$ (among others) hold considerable promise to constrain the abundance and properties of primordial black holes at increasing significance.

We are very grateful to D. Rubin for conversations and providing us with the Union outlier sample, L. Galbany for an appraisal of these outliers and the authors of Ref. [45] for the CMB data in Fig. 3, as well as G. Aldering, T. Collett, D. Goldstein, J. Guy, B. Hayden, D. Holz, T. de Jaeger, D. Jones, D. Kasen, A. Kim, E. Mediavilla, B. Metcalf, D. Scolnic, and S. Perlmutter for useful discussions. M. Z. is supported by the Marie Sklodowska-Curie Global Fellowship Project NLO-CO. This research used resources of the National Energy Research Scientific Computing Center, a DOE Office of Science User Facility supported by the Office of Science of the U.S. Department of Energy under Contract No. DE-AC02-05CH11231.

"miguelzuma@berkeley.edu

tuseljak@berkeley.edu

[1] P. A. R. Ade et al. (Planck Collaboration), Astron. Astrophys. 594, A13 (2016).

[2] G. Bertone, D. Hooper, and J. Silk, Phys. Rep. 405, 279 (2005).

[3] Y. B. Zeldovich and I. D. Novikov, Sov. Astron. 10, 602 (1967).

[4] B. J. Carr and S. W. Hawking, Mon. Not. R. Astron. Soc. 168, 399 (1974).

[5] P. Meszaros, Astron. Astrophys. 37, 225 (1974).

[6] G. F. Chapline, Nature (London) 253, 251 (1975).

[7] B. Carr, F. Kuhnel, and M. Sandstad, Phys. Rev. D 94, 083504 (2016).

[8] M. Sasaki, T. Suyama, T. Tanaka, and S. Yokoyama, Classical Quantum Gravity 35, 063001 (2018).

[9] B. Carr and J. Silk, Mon. Not. R. Astron. Soc. 478, 3756 (2018).

[10] B. P. Abbott et al. (Virgo, LIGO Scientific Collaborations), Phys. Rev. Lett. 116, 061102 (2016).

[11] B. P. Abbott et al. (Virgo, LIGO Scientific Collaborations), Phys. Rev. X 6, 041015 (2016).

[12] S. Bird, I. Cholis, J.B. Muñoz, Y. Ali-Haïmoud, M. Kamionkowski, E. D. Kovetz, A. Raccanelli, and A.G. Riess, Phys. Rev. Lett. 116, 201301 (2016).

[13] S. Clesse and J. García-Bellido, Phys. Dark Universe 15, 142 (2017).

[14] T. Nakamura, M. Sasaki, T. Tanaka, and K. S. Thorne, Astrophys. J. 487, L139 (1997).

[15] M. Sasaki, T. Suyama, T. Tanaka, and S. Yokoyama, Phys. Rev. Lett. 117, 061101 (2016).

[16] Y. Ali-Haïmoud, E. D. Kovetz, and M. Kamionkowski, Phys. Rev. D 96, 123523 (2017).

[17] Z.-C. Chen and Q.-G. Huang, arXiv:1801.10327.

[18] R. Magee, A.-S. Deutsch, P. McClincy, C. Hanna, C. Horst, D. Meacher, C. Messick, S. Shandera, and M. Wade, arXiv: 1808.04772.

[19] B. P. Abbott et al., arXiv:1808.04771.
[20] R. B. Metcalf and J. Silk, Phys. Rev. Lett. 98, 071302 (2007); 98, 099903(E) (2007).

[21] K. P. Rauch, Astrophys. J. 374, 83 (1991).

[22] R. B. Metcalf and J. Silk, Astrophys. J. 519, L1 (1999).

[23] U. Seljak and D. E. Holz, Astron. Astrophys. 351, L10 (1999).

[24] See Supplemental Material at http://link.aps.org/ supplemental/10.1103/PhysRevLett.121.141101, Secs. IA, IB for details on lensing by compact objects and the role of LSS, which include Refs. [25-34].

[25] C. C. Dyer and R. C. Roeder, Astrophys. J. 180, L31 (1973).

[26] S. Das and J. P. Ostriker, Astrophys. J. 645, 1 (2006).

[27] D.E. Holz and R. M. Wald, Phys. Rev. D 58, 063501 (1998).

[28] K. Kainulainen and V. Marra, Phys. Rev. D 80, 123020 (2009).

[29] K. Kainulainen and V. Marra, Phys. Rev. D 83, 023009 (2011).

[30] R. M. Quimby, M. Oguri, A. More, S. More, T. J. Moriya, M. C. Werner, M. Tanaka, G. Folatelli, M. C. Bersten, and K. Nomoto, Science 344, 396 (2014).

[31] A. Goobar et al., Science 356, 291 (2017).

[32] D. Rubin et al., arXiv:1707.04606.

[33] T. Castro and M. Quartin, Mon. Not. R. Astron. Soc. 443, L6 (2014).

[34] E. Macaulay, T. M. Davis, D. Scovacricchi, D. Bacon, T. E. Collett, and R. C. Nichol, Mon. Not. R. Astron. Soc. 467, 259 (2017).

[35] D. A. Goldstein, P.E. Nugent, D. N. Kasen, and T.E. Collett, Astrophys. J. 855, 22 (2018).

[36] Y. C. Pei, Astrophys. J. 403, 7 (1993).

[37] See Supplemental Material at http://link.aps.org/ supplemental/10.1103/PhysRevLett.121.141101, Sec. IC for details on finite-source effects in the PDF and PBH constraints, which includes Refs. [38-40].

[38] P. Schneider, Astron. Astrophys. 179, 71 (1987).

[39] M. Vietri and J. P. Ostriker, Astrophys. J. 267, 488 (1983).

[40] C. T. Byrnes, M. Hindmarsh, S. Young, and M. R. S. Hawkins, arXiv:1801.06138.

[41] P. Tisserand et al. (EROS-2 Collaboration), Astron. Astrophys. 469, 387 (2007).

[42] T. D. Brandt, Astrophys. J. 824, L31 (2016).

[43] T. S. Li et al. (DES), Astrophys. J. 838, 8 (2017).

[44] Y. Ali-Haïmoud and M. Kamionkowski, Phys. Rev. D 95, 043534 (2017).

[45] J. L. Bernal, N. Bellomo, A. Raccanelli, and L. Verde, J. Cosmol. Astropart. Phys. 10 (2017) 052.

[46] M. Betoule et al. (SDSS Collaboration), Astron. Astrophys. 568, A22 (2014).

[47] N. Suzuki et al., Astrophys. J. 746, 85 (2012).

[48] S. Alam et al. (BOSS Collaboration), Mon. Not. R. Astron. Soc. 470, 2617 (2017).

[49] See Supplemental Material at http://link.aps.org/ supplemental/10.1103/PhysRevLett.121.141101, Secs. IIA, IIB for details on the likelihood and $\mathrm{SNe}$ population modeling, which include Refs. [50-52].

[50] J. T. Nielsen, A. Guffanti, and S. Sarkar, Sci. Rep. 6, 35596 (2016).

[51] D. Rubin and B. Hayden, Astrophys. J. Lett. 833, L30 (2016). 
[52] S. M. Feeney, D. J. Mortlock, and N. Dalmasso, Mon. Not. R. Astron. Soc. 476, 3861 (2018).

[53] See Supplemental Material at http://link.aps.org/ supplemental/10.1103/PhysRevLett.121.141101, Sec. IIC for the complete discussion of our base analysis, which includes Refs. [54-56].

[54] D. Foreman-Mackey, D. W. Hogg, D. Lang, and J. Goodman, Publ. Astron. Soc. Pac. 125, 306 (2013).

[55] A. Lewis and S. Bridle (2013).

[56] J. Garcia-Bellido, S. Clesse, and P. Fleury, Phys. Dark Universe 20, 95 (2018).

[57] S. Taubenberger, The Extremes of Thermonuclear Supernovae, Handbook of Supernovae (Springer International Publishing AG, New York, 2017), p. 317, ISBN978-3-31921845-8.

[58] See Supplemental Material at http://link.aps.org/ supplemental/10.1103/PhysRevLett.121.141101, Sec. IIIA for the discussion of SNe outliers, which includes Refs. [59-72].

[59] A. V. Filippenko, M. W. Richmond, T. Matheson, J. C. Shields, E. M. Burbidge, R. D. Cohen, M. Dickinson, M. A. Malkan, B. Nelson, J. Pietz, D. Schlegel, P. Schmeer, H. Spinrad, C. C. Steidel, H. D. Tran, and W. Wren, Astrophys. J. 384, L15 (1992).

[60] M. M. Phillips, L. A. Wells, N. B. Suntzeff, M. Hamuy, B. Leibundgut, R. P. Kirshner, and C. B. Foltz, Astron. J. 103, 1632 (1992).

[61] D. A. Howell et al. (SNLS Collaboration), Nature (London) 443, 308 (2006).

[62] R. Scalzo et al. (Nearby Supernova Factory), Astrophys. J. 757, 12 (2012).

[63] M. A. Hamuy et al., Nature (London) 424, 651 (2003).

[64] B. Dilday et al., Science 337, 942 (2012).

[65] J. M. Silverman et al., Astrophys. J. Suppl. Ser. 207, 3 (2013).

[66] A. Conley et al. (SNLS Collaboration), Astrophys. J. Suppl. Ser. 192, 1 (2011).

[67] M. Kunz, B. A. Bassett, and R. A. Hlozek, Phys. Rev. D 75, 103508 (2007).

[68] M. Foxley-Marrable, T. E. Collett, G. Vernardos, D. A. Goldstein, and D. Bacon, Mon. Not. R. Astron. Soc. 478, 5081 (2018).

[69] D. Rubin et al. (Supernova Cosmology Project), Astrophys. J. 813, 137 (2015).

[70] R. J. Foley et al., Astron. J. 137, 3731 (2009).

[71] M. Ganeshalingam et al., Astrophys. J. 751, 142 (2012).

[72] C. Zheng et al. (SDSS-II Collaboration), Astron. J. 135, 1766 (2008).
[73] See Supplemental Material at http://link.aps.org/ supplemental/10.1103/PhysRevLett.121.141101, Secs. IIIB, IIIC for the discussion of correlated noise, selection effects and $\mathrm{SNe}$ evolution.

[74] E. Mediavilla, J. Jiménez-Vicente, J. A. Muñoz, H. VivesArias, and J. Calderón-Infante, Astrophys. J. 836, L18 (2017).

[75] Ł. Wyrzykowski, S. Kozłowski, J. Skowron, A. Udalski, M. K. Szymański, M. Kubiak, G. Pietrzyński, I. Soszyński, O. Szewczyk, K. Ulaczyk, and R. Poleski, Mon. Not. R. Astron. Soc. 413, 493 (2011).

[76] J. M. Diego et al., Astrophys. J. 857, 25 (2018).

[77] T. Venumadhav, L. Dai, and J. Miralda-Escud, Astrophys. J. 850, 49 (2017).

[78] M. Oguri, J. M. Diego, N. Kaiser, P. L. Kelly, and T. Broadhurst, Phys. Rev. D 97, 023518 (2018).

[79] D. Gaggero, G. Bertone, F. Calore, R. M. T. Connors, M. Lovell, S. Markoff, and E. Storm, Phys. Rev. Lett. 118, 241101 (2017).

[80] A. Hektor, G. Htsi, L. Marzola, M. Raidal, V. Vaskonen, and H. Veerme, Phys. Rev. D 98, 023503 (2018).

[81] K. Schutz and A. Liu, Phys. Rev. D 95, 023002 (2017).

[82] V. Poulin, P. D. Serpico, F. Calore, S. Clesse, and K. Kohri, Phys. Rev. D 96, 083524 (2017).

[83] M. Ricotti and A. Gould, Astrophys. J. 707, 979 (2009).

[84] G. Aslanyan, L. C. Price, J. Adams, T. Bringmann, H. A. Clark, R. Easther, G. F. Lewis, and P. Scott, Phys. Rev. Lett. 117, 141102 (2016).

[85] D. M. Scolnic et al., Astrophys. J. 859, 101 (2018).

[86] D. O. Jones et al., Astrophys. J. 857, 51 (2018).

[87] R. Kessler and D. Scolnic, Astrophys. J. 836, 56 (2017).

[88] S. Dhawan, A. Goobar, and E. Mrtsell, J. Cosmol. Astropart. Phys. 07 (2018) 024.

[89] S. Clesse and J. García-Bellido, Phys. Dark Universe 18, 105 (2017).

[90] E. D. Kovetz, I. Cholis, P. C. Breysse, and M. Kamionkowski, Phys. Rev. D 95, 103010 (2017).

[91] I. Cholis, E. D. Kovetz, Y. Ali-Haïmoud, S. Bird, M. Kamionkowski, J. B. Muñoz, and A. Raccanelli, Phys. Rev. D 94, 084013 (2016).

[92] M. Raidal, V. Vaskonen, and H. Veerme, J. Cosmol. Astropart. Phys. 09 (2017) 037.

[93] J. B. Muñoz, E. D. Kovetz, L. Dai, and M. Kamionkowski, Phys. Rev. Lett. 117, 091301 (2016).

[94] K. Van Tilburg, A.-M. Taki, and N. Weiner, J. Cosmol. Astropart. Phys. 07 (2018) 041.

[95] B. Horowitz, arXiv:1612.07264.

[96] T. Nakama, B. Carr, and J. Silk, Phys. Rev. D 97, 043525 (2018). 